\title{
THE SLING SHOT INCREASED THE MAXIMUM NUMBER OF REPETITIONS IN THE BARBELL BENCH PRESS IN MEN WITH DIFFERENT RESISTANCE TRAINING EXPERIENCE
}

original paper

(1) University School of Physical Education in Wroclaw

DOI: https://doi.org/10.5114/hm.2020.88150

\author{
GUSTAVO FERREIRA PEDROSA ${ }^{1,2}$, BRUNO VICTOR CORRÊA DA SILVA ${ }^{2,3}$, \\ GISELE FREIRE DA SILVA ${ }^{2,3}$, MARCOS HENRIQUE RODRIGUES DOS SANTOS ${ }^{3}$, \\ MARINA GURGEL SIMÕES ${ }^{2}$, GUILHERME SILVA ALVES FERREIRA ${ }^{4}$, \\ RICARDO FERREIRA PEDROSA ${ }^{3}$, RONALDO ÂNGELO DIAS DA SILVA ${ }^{2}$, \\ RODRIGO CÉSAR RIBEIRO DINIZ ${ }^{2}$ \\ ${ }^{1}$ Brazilian Air Force, Aeronautical Instruction and Adaptation Centre, Lagoa Santa, Brazil \\ ${ }^{2}$ Sports Department, Federal University of Minas Gerais, Belo Horizonte, Brazil \\ ${ }^{3}$ Physical Education Department, University Center of Belo Horizonte, Belo Horizonte, Brazil \\ ${ }^{4}$ Physical Education Department, University Center Estácio of Belo Horizonte, Belo Horizonte, Brazil
}

\begin{abstract}
Purpose. The study examined if the elastic device named Sling Shot could increase the maximum number of repetitions $(\mathrm{MNR})$ and diminish the mean repetition duration in men with different resistance training experience while performing the bench press exercise in multiple sets.

Methods. Overall, 22 men were grouped depending on their resistance training experience. The most experienced group (MEG; 11 men, $65.45 \pm 26.27$ months of training experience) and the less experienced group (LEG; 11 men, $3.09 \pm 2.07$ months of training experience) performed 3 sets at $80 \%$ of the 1-repetition maximum test as fast as possible, with 2 -min rest, of the barbell bench press exercise with and without the Sling Shot. Two 3-way ANOVA tests, with $\alpha=0.05$, were used to compare the MNR and mean repetition duration in inter- and intra-group comparisons across the sets.

Results. The Sling Shot increased the MNR in the 2 groups throughout the 3 sets. The increase was 50.5\%, 65.4\%, and $43.8 \%$ in the MEG group and $120 \%, 68.4 \%$, and $43.3 \%$ in the LEG group for the $1^{\text {st }}, 2^{\text {nd }}$, and $3^{\text {rd }}$ sets, respectively. However, there was no difference in the MNR between groups when the Sling Shot was used. Additionally, both groups performed the repetitions with a shorter mean duration with the Sling Shot than without it. No difference was observed between the groups. Conclusions. Regardless of training experience, the Sling Shot constitutes an alternative for increasing the MNR and decreasing the mean repetition duration in multiple sets.
\end{abstract}

Key words: training volume, repetition duration, facilitation strategy, performance

\section{Introduction}

The barbell bench press is a multi-articular exercise; it constitutes 1 of the 3 exercises from the powerlifting (squat, deadlift, and bench press) [1]. The exercise demands the work of the pectoralis major, anterior deltoid, and triceps brachii as primary muscles [2]. In addition, it has been frequently used to compare the physiological [3, 4] and mechanical responses [5, 6], as well as to test the effect of different strategies that aim for improving the physical performance [7-9]. Strategies such as chains [10] or elastic bands [11] have been applied to increase the load during the bench press exercise, and results that support their use have been presented. However, studies that investigated strategies aimed to make the completion of the barbell bench press exercise easier are rare. The facilitation of the barbell bench press exercise could lead, for example, to a greater training intensity $[9,12,13]$ or a greater volume load [14]. Yet, to the best of our knowledge,

Correspondence address: Gustavo Ferreira Pedrosa, Federal University of Minas Gerais, Av. Presidente Antônio Carlos, nº627, CEP: 31270-901, Belo Horizonte, Brazil, e-mail: gustavofpedrosa@gmail.com

Received: December 25, 2018

Accepted for publication: July 7, 2019

Citation: Pedrosa GF, da Silva BVC, da Silva GF, dos Santos MHR, Simões MG, Ferreira GSA, Pedrosa RF, da Silva RAD, Diniz RCR. The sling shot increased the maximum number of repetitions in the barbell bench press in men with different resistance training experience. Hum Mov. 2020;21(1):22-31; doi: https://doi.org/10.5114/hm.2020.88150. 
only 3 studies have investigated a facilitation strategy consisting in the usage of a chest device called a Sling Shot $[9,13,14]$.

Ye et al. [13] and Dugdale et al. [9] included strengthtrained men and strength athletes, respectively, in a sample population to compare the maximum weight lifted during the 1-repetition maximum test (1-RM) with and without the Sling Shot. In both studies, heavier weights were lifted when the Sling Shot was used. Additionally, Dugdale et al. [9] evaluated the weight lifted in the 3-RM and 8-RM tests, noticing that a greater amount of weight was lifted with the Sling Shot. The results obtained by Ye et al. [13] and Dugdale et al. [9] showed that with the Sling Shot, the volunteers performed the 1-RM, 3-RM, and 8-RM tests with heavier weights as well as at higher speeds. However, these studies did not compare the maximum number of repetitions (MNR) or the mean repetition duration that could be performed with and without the Sling Shot at the same relative intensity. In a study by Niblock and Steele [14] that used maximum intensity, the volume load (weight lifted $\times$ number of repetitions) was compared in a single set with and without the Sling Shot. The results showed that a greater volume load could be performed when the Sling Shot was applied. However, the authors did not compare the number of repetitions performed between the conditions in submaximal intensities or across multiple sets. Considering the results observed by Niblock and Steele [14], owing to the greater volume load achieved with the Sling Shot than without the Sling Shot, perhaps a longer time would be necessary for recovery to keep the volume load found in the $1^{\text {st }}$ set if subsequent sets were performed [15]. Would MNR drop significantly if short intra-set rest periods were employed while using the Sling Shot? In addition, different submaximal intensities may also alter the MNR performed [16, 17]. It is unknown whether the use of the Sling Shot would allow an individual to perform a greater number of repetitions than without the Sling Shot in submaximal intensity and in multiple sets. A greater MNR performed can be associated with higher muscle endurance [18], which is an important factor for performance in some sports [19]. In addition, as repetition duration is a factor that may influence the MNR, even with a determined relative intensity [20], the duration must be considered in the analysis as being relevant in the comparison of the MNR between different conditions (i.e. with and without the Sling Shot). Another factor that needs attention is the difference in the strength performance between trained and untrained men [16, 17].
Studies that have evaluated the MNR between individuals with different training experience show little [17] or no influence [16] of this variable. However, these studies have analysed the MNR after a single set, which may not represent the response for multiple sets. As discussed by Cormie et al. [21], central adaptations such as improvement of the motor unit recruitment, firing frequency, and muscle synchronisation can all be optimised by resistance training, and these factors can be linked to a greater performance over time. As a result, increases in glycogen and phosphocreatine levels are expected in response to strength training [22]. These responses represent an augmentation in the energy stock that could lead to better performance [22]. In addition, training experience may promote a hypertrophic effect, and thus, the addition of the contractile proprieties of the muscle may improve strength performance [23]. As these cited adaptations interfere with the process of force production and its respective maintenance during exercise [21, 22], it is possible that more repetitions over multiple sets could be performed by individuals with more training experience than individuals with less experience.

Considering the reasoning cited above for the comparison of the MNR between men with different training experience, it is possible that the advantage of using the Sling Shot may not be greater in more experienced men. This rationale includes the fact that the elastic force contribution from the Sling Shot during the repetitions should be the same for all subjects, regardless of training experience (under equal conditions). In this case, more experienced men may perform more repetitions with the Sling Shot than less experienced ones owing to the central and peripheral adaptations that occur across the training experience and not because of the Sling Shot facilitation. Regardless of the training experience, as the Sling Shot contributes to accelerating the movement $[9,13]$, the mean repetition duration may possibly be shorter when the Sling Shot is used.

Thus, the objective of the study was to compare the MNR and the mean repetition duration between individuals with different training experience with and without the Sling Shot in multiple sets. We hypothesised that in all sets, the use of the Sling Shot would allow the performance of a greater number of repetitions of shorter duration regardless of training experience. With a similar contribution from the Sling Shot, it was expected that individuals with greater experience would perform a greater number of repetitions than those with less experience. 


\section{Material and methods}

\section{Experimental design}

The study compared the MNR and the mean repetition duration in the barbell bench press exercise between 2 groups of men with different training experience, across 3 sets with or without the Sling Shot. Each group was subjected to 3 experimental sessions: session 1, for familiarisation with the 1-RM test; session 2, performance of the 1-RM test; and session 3, a randomised selection involving the completion of the MNR with or without the Sling Shot. The sessions were separated by 48 hours, and the time of data collection was maintained for each subject across all sessions. Figure 1 shows the experimental design of the study.

\section{Sample}

The sample size was calculated from the data of Ye et al. [13], who also used the Sling Shot. In this study, it was found that $114.6 \pm 18.6 \mathrm{~kg}$ and $132.1 \pm 18.5 \mathrm{~kg}$ were lifted in the bench press exercise with and without the Sling Shot, respectively, which generated an effect size of 0.94 (Cohen's $d$ ). This value, when inserted in the $G^{*}$ Power software (version 3.1.9.2; Heinrich

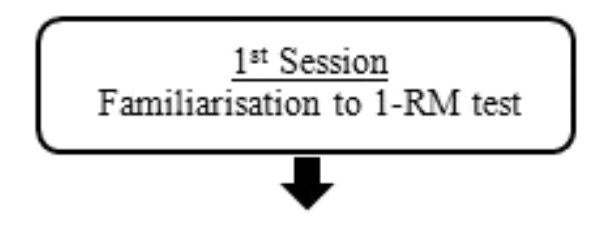

48-hours rest

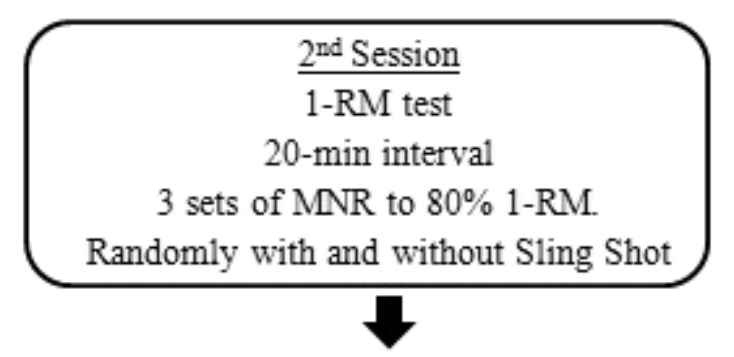

48-hours rest

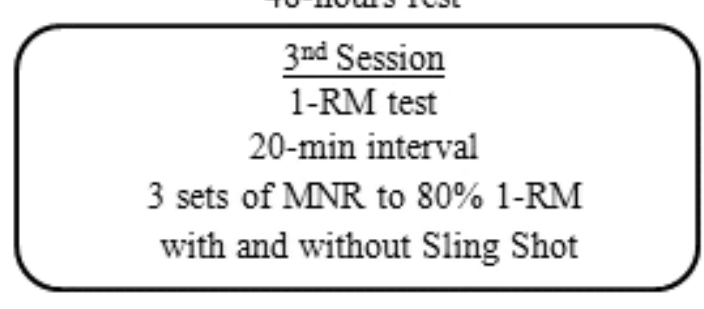

1-RM test - 1-repetition maximum test

MNR - maximum number of repetitions

Figure 1. Experimental design
Heine University Düsseldorf, Germany) with a power of 0.90 and $\alpha=0.05$, provided the number of 16 participants. Considering possible sample loss, we invited 22 volunteers to participate in the study. Therefore, 22 healthy adult men who were already enrolled in resistance training programs and had no history of injury entered the study. They were assigned to 2 groups depending on their training experience. The most experienced group (MEG) was composed of 11 men with at least 3 years of continuous experience in the barbell bench press exercise. The other group, the less experienced group (LEG), consisted of 11 men with 1-6 months of experience in the barbell bench press exercise. The subjects' training routines were modified during the data collection period, and the volunteers were told not to perform exercises that required efforts of the pectoralis major, anterior deltoid, or triceps brachii muscles during the 48 hours prior to each session. Furthermore, each subject was instructed not to perform any physical activity before the experimental sessions and to maintain their dietary practices. In addition, all volunteers were informed about the objectives and methods of the experiment. Table 1 presents detailed information on the 2 groups.

\section{Experimental sessions 1, 2, and 3}

First, the anthropometric characteristics were measured. Immediately afterwards, the hand position on the bar was determined in accordance with the individual's preference. The bar was numerically marked along its length with adhesive strips that remained glued throughout the study. Thus, in the $1^{\text {st }}$ experimental session, the volunteers identified the hand position and one of the researchers recorded the grip locations, which allowed the exact reproduction of the grip throughout the sessions and sets. The position of the head on the bench was also standardised by a numerical tape affixed on the side along the bench press (Vitally Convergent, São José do Rio Preto, Brazil). When the subject lay on the bench for the first time, one of the researchers recorded the number that indicated the position of the head to be used for later reproduction.

Subsequently, the participants were familiarised with the 1-RM test for the barbell bench press exercise. The familiarisation and the actual 1-RM test were performed during the $1^{\text {st }}$ and $2^{\text {nd }}$ sessions ( 48 hours apart), which aimed to familiarise the subjects with the procedures and to define the weight for the following phases, respectively. As the familiarisation and the actual 1-RM test followed the same procedures, 
Table 1. Group profiles (mean \pm standard deviation)

\begin{tabular}{ccccccr}
\hline $\begin{array}{c}\text { Group } \\
(n=22)\end{array}$ & $\begin{array}{c}\text { Age } \\
(\text { years })\end{array}$ & $\begin{array}{c}\text { Body mass } \\
(\mathrm{kg})\end{array}$ & $\begin{array}{c}\text { Height } \\
(\mathrm{cm})\end{array}$ & $\begin{array}{c}\text { Body fat } \\
(\%)\end{array}$ & $\begin{array}{c}\text { Length of } \\
\text { experience } \\
(\text { months })\end{array}$ & $\begin{array}{c}\text { 1-RM } \\
(\mathrm{kg})\end{array}$ \\
\hline MEG $(n=11)$ & $29.18 \pm 5.54$ & $83.34 \pm 13.41$ & $178.32 \pm 6.59$ & $11.26^{*} \pm 5.07$ & $65.45^{*} \pm 26.27$ & $106.70^{*} \pm 9.20$ \\
LEG $(n=11)$ & $27.64 \pm 4.81$ & $78.51 \pm 5.37$ & $176.50 \pm 3.56$ & $18.91 \pm 8.35$ & $3.09 \pm 2.07$ & $73.70 \pm 11.93$ \\
\hline
\end{tabular}

MEG - most experienced group, LEG - less experienced group, 1-RM - one repetition maximum test for barbell bench press exercise

* difference of the MEG group ( $p<0.05$, Student's $t$-test)

we are going to explain only the test. The test began with an eccentric muscle action by lowering the bar to the 1-RM sternum, followed by a concentric muscle action, determined by elbow extension. The 1-RM test was concluded within a maximum of 5 attempts [24], with 5-min rest periods between them. For each attempt, there was a minimum increase of $2 \mathrm{~kg}$ onto the bar. When the volunteer could not perform a complete repetition (full range of motion), the prior weight lifted was recorded as representing the 1-RM test result. To establish the correlation between the greater weight lifted in the familiarisation and in the 1-RM test, the intra-class correlation coefficient $\left(\operatorname{ICC}_{[3, \mathrm{k}]}\right)$ was used, which presented values equal to 0.99 and 0.98 for the MEG and LEG groups, respectively, demonstrating consistency between the 2 measures and reliability of the 1-RM test result. In addition, during the week prior to the experimental sessions, all subjects performed the barbell bench press exercise with the Sling Shot on at least 3 different days (3 sets of 8 ca. 12-RM each day) to familiarise themselves with the device and to select 1 of the 2 Sling Shot sizes available (large: $31.75 \mathrm{~cm}$; X-large: $36.83 \mathrm{~cm}$ ). During all data collection, a minimum of 2 researchers with more than 3 years of resistance training experience evaluated the execution of the movements and the recording of the data.

After the 1-RM test, the participants rested for 20 minutes [25]. Then, they were positioned on the barbell bench press, and the weight that represented $80 \%$ of the weight found in the 1-RM test was inserted onto the bar. Subsequently, the volunteers randomly performed the MNR in 3 sets until concentric failure in the highest speed possible (verbal encouragement) with and without the Sling Shot (randomly selected). At the concentric failure, the set was interrupted, and the last complete repetition was recorded. Between sets, a 2-min rest was offered. In addition, the time under tension of each set was recorded with a manual stopwatch, which was triggered at the beginning of the eccentric action and stopped at the time of the concentric failure in each set. The time under tension of the set was divided by the MNR performed to estimate the mean repetition duration across the set, making it possible to compare the mean repetition duration between the groups and between the conditions with or without the Sling Shot. Two days later, the subjects performed the MNR with the same procedures as previously used but, this time, with or without the Sling Shot (in accordance with the randomised selection procedures). The Sling Shot was placed in a way different than that reported by Dugdale et al. [9]. In the present study, it was located at the armpit level, surrounding the arms (Figure 2). The $80 \%$ intensity and the 2-min rest were chosen to follow the training protocols designed for the development of strength and hypertrophy [26].

\section{Statistical analysis}

Initially, a descriptive analysis of the data was performed. The normality and homogeneity of variances were verified by using Shapiro-Wilk and Mauchly's

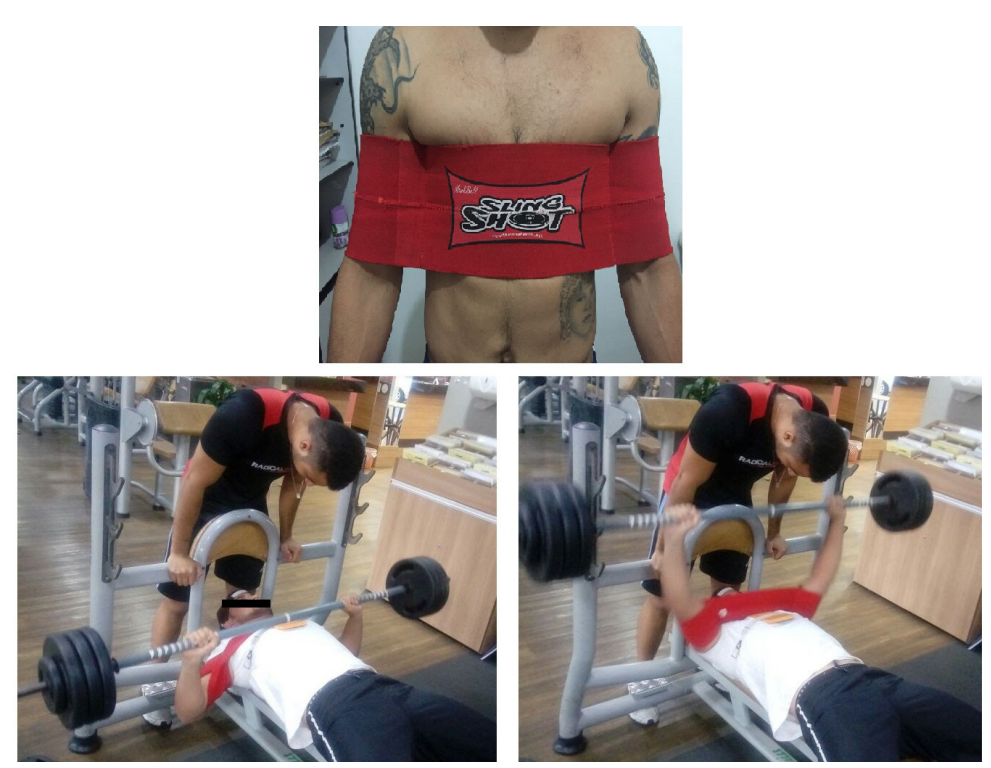

Figure 2. Sling Shot placement and exercise execution 
tests, respectively. To compare the mean repetition duration and the MNR within and between groups across the sets, with and without the Sling Shot, two 3-way ANOVA tests were used (factor 1: group [MEG and LEG]; factor 2: condition [with and without the Sling Shot]; and factor 3 : set $\left[1^{\text {st }}, 2^{\text {nd }}\right.$, and $3^{\text {rd }}$ set]). In addition, to determine how much (in percentages) the Sling Shot contributed to MNR increase in within- and between-group comparisons across the sets, a 2-way ANOVA test was applied (factor 1: group [MEG and LEG]; and factor 2 : set [ $1^{\text {st }}, 2^{\text {nd }}$, and $3^{\text {rd }}$ set]). To establish such comparisons, the differences in the MNR performed with and without the Sling Shot in each group were calculated as percentages. When necessary, the Bonferroni post-hoc test was selected to identify the differences found by the ANOVAs. The level of significance adopted was $\alpha=0.05$. In addition, eta squared $\left(\eta^{2}\right)$ values were reported to reflect the magnitude of the differences in each treatment as indicative of the effect size (small: 0.01, medium: 0.06, and large: 0.14) [27]. All statistical procedures were performed with the SPSS software (IBM SPSS 22.0; IBM, Armonk, USA).

\section{Ethical approval}

The research related to human use has complied with all the relevant national regulations and institutional policies, has followed the tenets of the Declaration of Helsinki, and has been approved by the authors' institutional review board or an equivalent committee.

\section{Informed consent}

Informed consent has been obtained from all individuals included in this study.

\section{Results}

\section{Mean repetition duration}

When comparing the mean repetition duration, the 3-way ANOVA found only main effects, for condition $\left(F_{1}=0.001, p=0.001, \eta^{2}=0.25\right.$, power $\left.=0.98\right)$ and for set $\left(F_{2}=20.479, p<0.001, \eta^{2}=0.12\right.$, power $=0.98)$. No interaction between factors was found. Regarding the main effect for condition, the repetitions were performed in a shorter mean duration with the Sling Shot than without it. For the main effect for set, the $3^{\text {rd }}$ set was performed with longer mean repetition duration than the $1^{\text {st }}$ and $2^{\text {nd }}$ sets. Similarly, the $2^{\text {nd }}$ set was performed with a longer mean repetition duration than the $1^{\text {st }}$ set. Figure 3 shows the mean repetition duration across the sets for all groups and conditions.

\section{Maximum number of repetitions}

In the comparison of the MNR, the 3-way ANOVA found a triple interaction effect: between groups, conditions, and sets $\left(F_{2}=3.507, p=0.04, \eta^{2}=0.02\right.$, power $>0.99)$. The post-hoc analysis showed that both groups performed similarly more repetitions with the Sling Shot than without the Sling Shot across the 3 sets. In addition, with the Sling Shot, both groups performed more repetitions in the $1^{\text {st }}$ set than they did in the $2^{\text {nd }}$ and $3^{\text {rd }}$ sets, and they performed more repetitions in the $2^{\text {nd }}$ set than during the $3^{\text {rd }}$ set. Without the Sling Shot, the MEG group performed a greater number of repetitions than the LEG group in the $1^{\text {st }}$ set, and both the MEG and LEG groups performed a similar number of repetitions between the $2^{\text {nd }}$ and $3^{\text {rd }}$ sets. Without the Sling Shot and within each group, the MEG group performed more repetitions in the $1^{\text {st }}$ set than they did in the $2^{\text {nd }}$ and $3^{\text {rd }}$ sets, and in the $2^{\text {nd }}$ set, more repetitions were performed than during the $3^{\text {rd }}$ set. For the LEG group, a similar number of repetitions were performed in the $1^{\text {st }}$ and $2^{\text {nd }}$ sets, and in these 2 sets, the subjects performed a greater number of repetitions than they did in the $3^{\text {rd }}$ set. Figure 4 shows the MNR across the sets for all groups and conditions.

Regarding the percentage of how much the Sling Shot contributed to increasing the MNR between and within the groups across sets, the 2-way ANOVA showed an interaction effect between groups and sets $\left(F_{2}=\right.$ 34.15, $p=0.02, \eta^{2}=0.04$, power $\left.>0.61\right)$. The Bonferroni post-hoc test demonstrated that for the $1^{\text {st }}$ set, the Sling Shot contributed to the performance of more repetitions in the LEG group than in the MEG group. In addition, for the LEG group, the percentage of contribution from the Sling Shot was greater in the $1^{\text {st }}$ set than in the $3^{\text {rd }}$ set, and there was no difference in the percentage of contribution from the Sling Shot across the sets in the MEG group. Figure 5 shows the percentage of contribution from the Sling Shot in both groups across the sets.

\section{Discussion}

The aim of this study was to compare both the MNR at $80 \%$ of 1-RM in the barbell bench press exercise in multiple sets with and without the Sling Shot in 2 different training experience groups (MEG and LEG) and the mean repetition duration between groups across the sets. As the main result, a greater number of repetitions were performed with the Sling Shot than without it, regardless of the group. In addition, there was no difference in the MNR between 
A) MEG

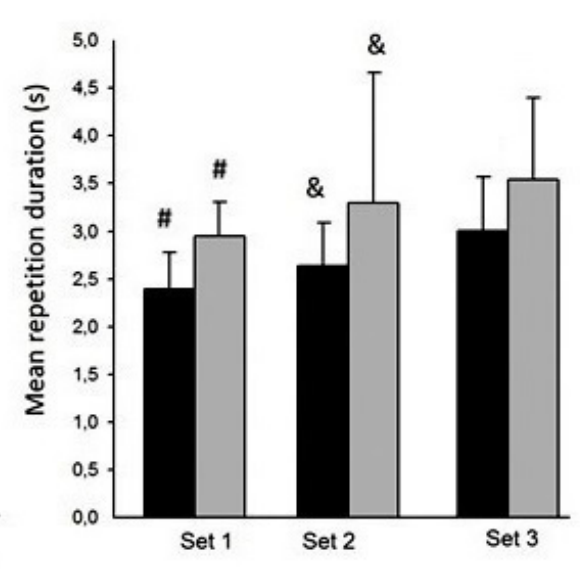

C) MEG $x$ LEG with Sling Shot

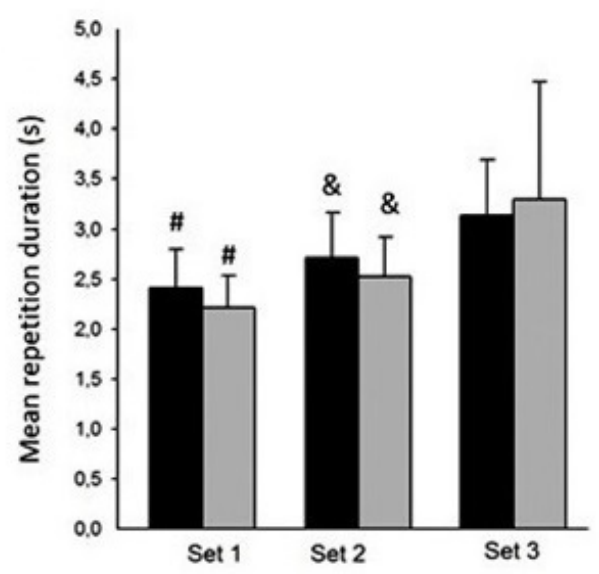

B) LEG

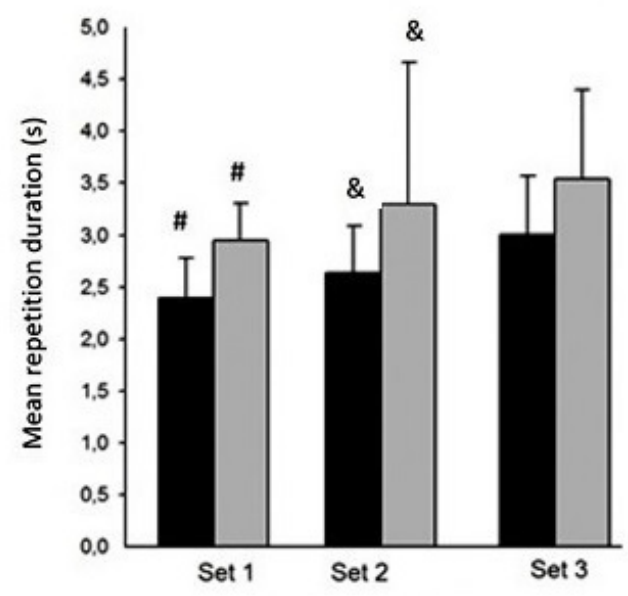

MEG LEG

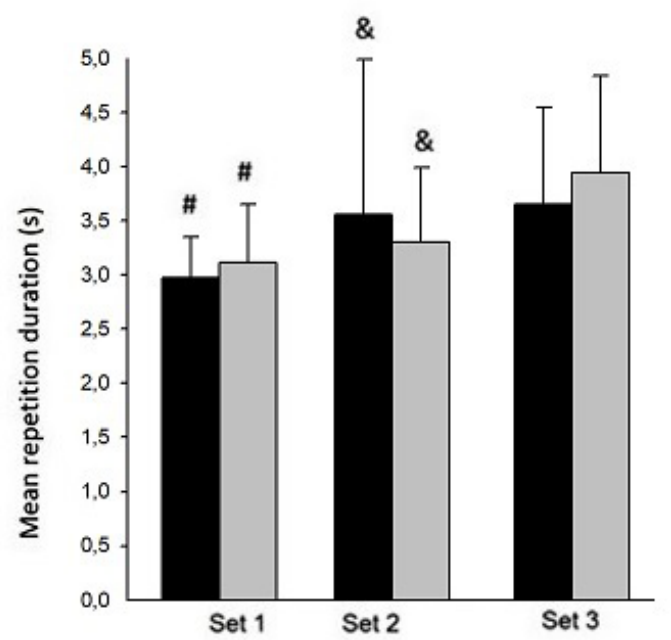

MEG - most experienced group, LEG - less experienced group Vertical lines denote standard errors.

\# different from the $2^{\text {nd }}$ and $3^{\text {rd }}$ set in the respective condition

$\&$ different from the $3^{\text {rd }}$ set in the respective condition, $p<0.05$

Figure 3. Mean repetition duration for MEG (A), LEG (B), MEG $\times$ LEG with Sling Shot (C), and MEG $\times$ LEG without Sling Shot $(\mathrm{D})$

the groups when the Sling Shot was used, contradicting what was expected. No difference was also observed between the groups in the mean repetition duration, regardless of the condition (with or without the Sling Shot) or the set.

In the present study, a greater number of repetitions were performed in all sets when the Sling Shot was used, regardless of the group. There are some potential factors that could explain this increase in the number of repetitions. Firstly, the external elastic force provided by the Sling Shot potentiates the stretch-shortening cycle during each repetition [28, 29]. However, it is unknown how much of this is due to the Sling Shot's mechanical stiffness because this information was not found in product specifications from the manufacturer or quantified in the present study or in previous investigations $[9,13,14]$. Nevertheless, in the earlier studies, the use of the Sling Shot caused an increase in the maximum weight lifted during the 1-RM test of ca. $16 \mathrm{~kg}$ [13] and ca. $20 \mathrm{~kg}$ [9], suggest- 


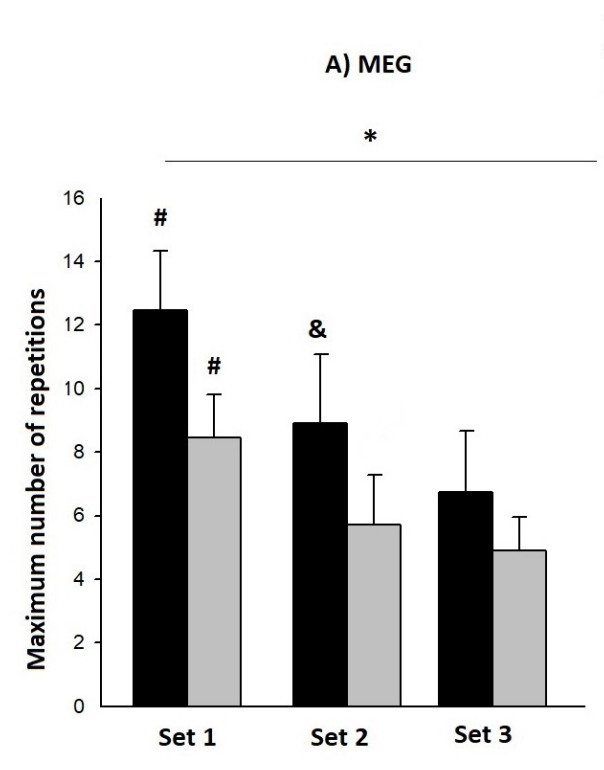

With Sling Shot

Without Sling Shot
B) LEG

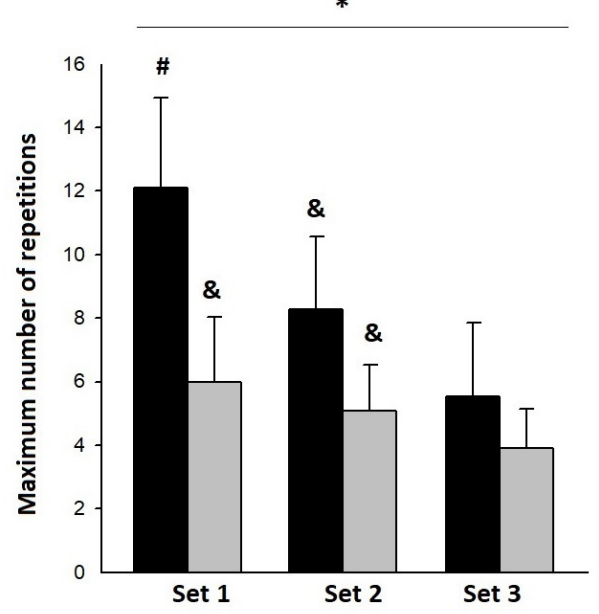

C) MEG $x$ LEG with Sling Shot

D) MEG $x$ LEG - without Sling Shot

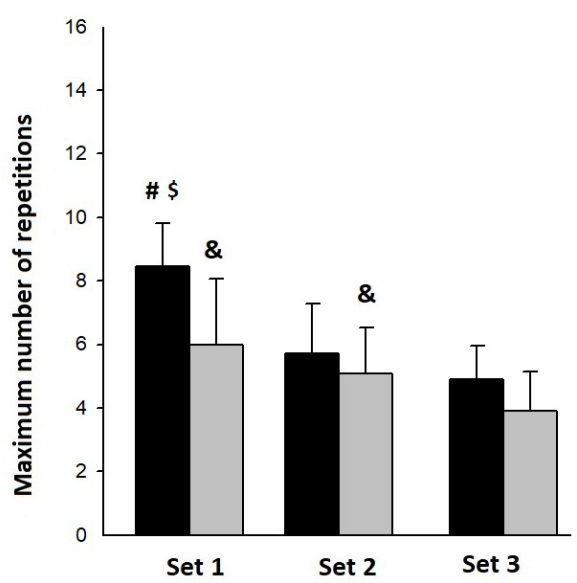

MEG - most experienced group, LEG - less experienced group Vertical lines denote standard errors.

* different between the conditions in all sets

$\$$ different than the $1^{\text {st }}$ set of the MEG group

\# different from the $2^{\text {nd }}$ and $3^{\text {rd }}$ set in the respective condition

$\&$ different from the $3^{\text {rd }}$ set in the respective condition, $p<0.05$

Figure 4. Maximum number of repetitions for MEG (A), LEG (B), MEG $\times$ LEG with Sling Shot (C), and MEG $\times$ LEG without Sling Shot $(\mathrm{D})$

ing an elastic assistance when the Sling Shot was applied. Additionally, it has been shown that the Sling Shot can increase the bar velocity [13]. Although this variable was not measured, which is a limitation of the study, the mean repetition duration was calculated, and it was significantly shorter when the Sling Shot was used, regardless of the group. Therefore, our findings support the reasoning that an increase in the bar velocity occurred because of the use of the
Sling Shot. These results are in accordance with previous studies which demonstrated that subjects were able to perform more repetitions at either higher speed [5] or shorter repetition duration [30]. It seems that the Sling Shot offers a greater contribution of elastic force at the beginning of the concentric action. Consequently, the muscular force to move the bar off the chest and overcome the sticking point [31] may have been less than the muscular force produced when 


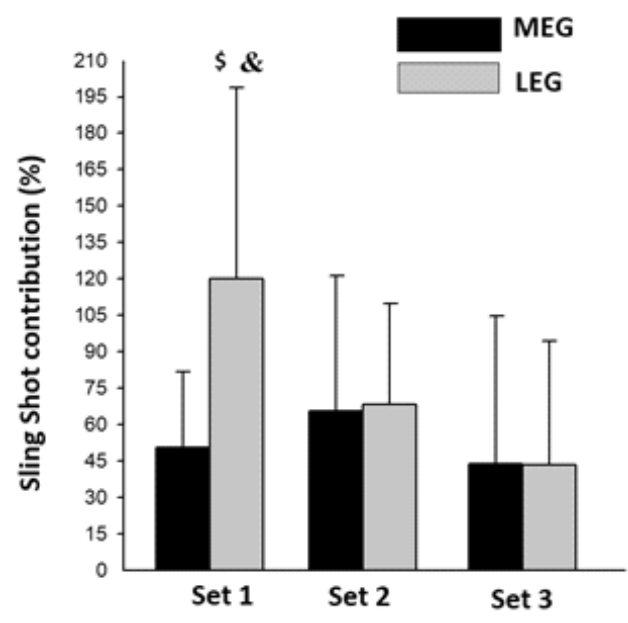

MEG - most experienced group, LEG - less experienced group Vertical lines denote standard errors.

$\$$ different than the $1^{\text {st }}$ set of the MEG group

$\&$ different from the $3^{\text {rd }}$ set in the respective condition, $p<0.05$

Figure 5. Contribution (\%) from Sling Shot to performance of more repetitions for MEG $\times$ LEG across the sets

the Sling Shot was not used at the same position, thus retarding the fatigue and enabling the participants to perform more repetitions.

Despite the groups performing a similar number of repetitions across the sets with the Sling Shot, a percentage analysis (Figure 4) showed that the Sling Shot contributed more in the LEG group than in the MEG group in the $1^{\text {st }}$ set. This increase was $50.5 \%$, $65.4 \%$, and $43.8 \%$ in the MEG group and $120 \%, 68.4 \%$, and $43.3 \%$ in the LEG group for the $1^{\text {st }}, 2^{\text {nd }}$, and $3^{\text {rd }}$ sets, respectively. The explanation of this finding could be linked to the central nervous system. Owing to greater training experience, it is expected that the subjects of the MEG group, in comparison with the LEG group, have greater central adaptation, such as corticospinal excitability [32], motor unit synchronisation [33], and a decrease in co-activation (antagonist muscle activation) [21], which could lead to greater motor control during an exercise they are accustomed to performing. However, with the Sling Shot, the individuals of the MEG group had to adjust their movement patterns that should be already well consolidated, to take advantage of the elastic contribution from the Sling Shot. On the contrary, because the individuals of the LEG group were less experienced than those in the MEG group, the motor adjustment to use the Sling Shot was probably easier for them to achieve. Thus, this group was aided in greater magnitude by the Sling Shot than the LEG group, even though both groups had performed the same MNR with the Sling Shot. This reasoning finds support in the comparison of the num- ber of repetitions between the LEG and MEG groups without the Sling Shot. As expected, the MEG group performed more repetitions than the LEG group in the $1^{\text {st }}$ set, which indicates that their experience in training may impact on the MNR when there is no requirement of motor adjustment during a known exercise such as the barbell bench press.

Concerning the reduction of the number of repetitions across the sets, it is possible that the 2-min rest was not enough to re-establish the central and peripheral conditions to produce the same initial force level [34]. This line of reasoning is reinforced by the reduction of the mean repetition duration across the sets. This reduction indicates that the speed at which the repetitions were performed diminished across the sets. Also, it has been reported that a reduction in the muscle shortening velocity can be associated with fatigue [35]. Although the use of the Sling Shot is oriented towards speed training, we also suggest its application aimed at a hypertrophic response. This is based on the fact that the volume and velocity moment seems to modulate the hypertrophic response [36]. In this sense, as we observed that the Sling Shot aided performance of more repetitions in less time (repetition duration), it is reasonable to propose its use for this approach. However, future experimental studies need to confirm this chronic effect on the hypertrophic response.

Another aspect that needs clarification is the possible placebo effect that may have occurred and influenced our data. In this case, more repetitions were probably performed not because of the elastic contribution of the Sling Shot but owing to the placebo effect. The presence of this effect could be checked by recruiting another experimental group that would be using a type of a placebo device (believing that it is the Sling Shot) while performing the repetitions through the sets. This placebo device could have the same design as the Sling Shot, but it would offer no elastic contribution. The lack of this third group is a potential limitation in our study, and future investigations should take this into account. In addition, in further studies using the Sling Shot, it would be interesting to register and present data regarding the Sling Shot curve length and the tension it could produce. This information could be useful for checking the calibration of the device.

\section{Conclusions}

In summary, this study showed that a greater number of repetitions were performed in the barbell bench 
press exercise with the Sling Shot than without the Sling Shot, regardless of the individuals' training experience. In addition, the repetitions were performed with a shorter mean repetition duration when the Sling Shot was used.

\section{Acknowledgments}

We would like to thank Carolina Augusta MedinaOliveira for the English language support.

\section{Disclosure statement}

No author has any financial interest or received any financial benefit from this research.

\section{Conflict of interest}

The authors state no conflict of interest.

\section{References}

1. Ryguła I, Płóciennik Ł, Lipińska P. Diagnostic sources of information on sports result determinants in young powerlifting athletes. Hum Mov. 2016;17(3):168-175; doi: 10.1515/humo-2016-0027.

2. Stastny P, Gołaś A, Blazek D, Maszczyk A, Wilk M, Pietraszewski P, et al. A systematic review of surface electromyography analyses of the bench press movement task. PLoS One. 2017;12(2):e0171632; doi: 10.1371/ journal.pone.0171632.

3. Lacerda LT, Martins-Costa HC, Diniz RCR, Lima FV, Andrade AGP, Tourino FD, et al. Variations in repetition duration and repetition numbers influence muscular activation and blood lactate response in protocols equalized by time under tension. J Strength Cond Res. 2016;30(1):251-258; doi: 10.1519/JSC.0000000 000001044.

4. Lacerda LT, Costa CG, Lima FV, Martins-Costa HC, Diniz RCR, Andrade AGP, et al. Longer concentric action increases muscle activation and neuromuscular fatigue responses in protocols equalized by repetition duration. J Strength Cond Res. 2019;33(6):1629-1639; doi: 10.1519/JSC.0000000000002148.

5. Sakamoto A, Sinclair PJ. Muscle activations under varying lifting speeds and intensities during bench press. Eur J Appl Physiol. 2012;112(3):1015-1025; doi: 10.1007/s00421-011-2059-0.

6. Sampson JA, Donohoe A, Groeller H. Effect of concentric and eccentric velocity during heavy-load non-ballistic elbow flexion resistance exercise. J Sci Med Sport. 2014;17(3):306-311; doi: 10.1016/j.jsams.2013.04.012.

7. Zemková E, Jeleň M, Ollé G, Vilman T, Hamar D. Power production during bench press with different ranges of motion on stable and unstable surfaces. Hum Mov. 2013;14(4):328-333; doi: 10.2478/humo-20130039.

8. Walker S, Hulmi JJ, Wernbom M, Nyman K, Kraemer WJ, Ahtiainen JP, et al. Variable resistance train- ing promotes greater fatigue resistance but not hypertrophy versus constant resistance training. Eur J Appl Physiol. 2013;113(9):2233-2244; doi: 10.1007/s00421 -013-2653-4.

9. Dugdale JH, Hunter AM, Di Virgilio TG, Macgregor LJ, Hamilton DL. Influence of the "Slingshot" bench press training aid on bench press kinematics and neuromuscular activity in competitive powerlifters. J Strength Cond Res. 2019;33(2):327-336; doi: 10.1519/JSC.00000000 00001853.

10. Ghigiarelli JJ, Nagle EF, Gross FL, Robertson RJ, Irrgang JJ, Myslinski T. The effects of a 7-week heavy elastic band and weight chain program on upper-body strength and upper-body power in a sample of division 1-AA football players. J Strength Cond Res. 2009;23(3): 756-764; doi: 10.1519/JSC.0b013e3181a2b8a2.

11. Bellar DM, Muller MD, Barkley JE, Kim CH, Ida K, Ryan EJ, et al. The effects of combined elastic- and free-weight tension vs. free-weight tension on one-repetition maximum strength in the bench press. J Strength Cond Res. 2011;25(2);459-463; doi: 10.1519/JSC.0b 013e3181c1f8b6.

12. Ahtiainen JP, Parkarinen A, Kraemer WJ, Häkkinen K. Acute hormonal responses heavy resistance exercise in strength athletes versus nonathletes. Can J Appl Physiol. 2004;29(5):527-543; doi: 10.1139/h04-034.

13. Ye X, Beck TW, Stock MS, Fahs CA, Kim D, Loenneke JP, et al. Acute effects of wearing an elastic, supportive device on bench press performance in young, resistancetrained males. Gazz Med Ital Arch Sci Med. 2014; 173(3):91-101.

14. Niblock J, Steele J. The 'Slingshot' can enhance volume-loads during performance of bench press using unaided maximal loads. J Trainol. 2017;6(2):47-51; doi: 10.17338/trainology.6.2_47.

15. Richmond SR, Godard MP. The effects of varied rest periods between sets to failure using the bench press in recreationally trained men. J Strength Cond Res. 2004;18(4):846-849; doi: 10.1519/14833.1.

16. Pedrosa G, Boaventura S, Abreu E, Gonçalves R, Prestes J, Gordon M, et al. Resistance exercise type but no training status affects the relationship between the maximum number of repetitions and the selected percentages of one repetition maximum. Gazz Med Ital Arch Sci Med. 2016;175(9):371-377.

17. Shimano T, Kraemer WJ, Spiering BA, Volek JS, Hatfield DL, Silvestre R, et al. Relationship between the number of repetitions and selected percentages of one repetition maximum in free weight exercises in trained and untrained men. J Strength Cond Res. 2006;20(4): 819-823; doi: 10.1519/R-18195.1.

18. Campos GE, Luecke TJ, Wendeln HK, Toma K, Hagerman FC, Murray TF, et al. Muscular adaptations in response to three different resistance-training regimens: specificity of repetition maximum training zones. Eur J Appl Physiol. 2002;88(1-2):50-60; doi: 10.1007/ s00421-002-0681-6. 
19. Bonitch-Góngora JG, Almeida F, Padial P, BonitchDomínguez JG, Feriche B. Maximal isometric handgrip strength and endurance differences between elite and non-elite young judo athletes. Arch Budo. 2013;9(4): 239-248.

20. Wilk M, Golas A, Stastny P, Nawrocka M, Krzysztofik M, Zajac A. Does tempo of resistance exercise impact training volume? J Hum Kinet. 2018;62:241-250; doi: 10.2478/hukin-2018-0034.

21. Cormie P, McGuigan MR, Newton RU. Developing maximal neuromuscular power: Part 1 - Biological basis of maximal power production. Sports Med. 2011;41(1): 17-38; doi: 10.2165/11537690-000000000-00000.

22. Booth FW, Thomason DB. Molecular and cellular adaptation of muscle in response to exercise: perspectives of various models. Physiol Rev. 1991;71(2):541585; doi: 10.1152/physrev.1991.71.2.541.

23. Noorkõiv M, Nosaka K, Blazevich AJ. Neuromuscular adaptations associated with knee joint angle-specific force change. Med Sci Sports and Exerc. 2014;46(8): 1525-1537; doi: 10.1249/MSS.0000000000000269.

24. Martins-Costa HC, Diniz RCR, Lima FV, Machado SC, Almeida RSV, Andrade AGP, et al. Longer repetition duration increases muscle activation and blood lactate response in matched resistance training protocols. Motriz Rev Educ Fis. 2016;22(1):35-41; doi: 10.1590/ S1980-65742016000100005.

25. Lagally KM, Robertson RJ, Gallagher KI, Goss FL, Jakicic JM, Lephart SM, et al. Perceived exertion, electromyography, and blood lactate during acute bouts of resistance exercise. Med Sci Sports Exerc. 2002;34(3): 552-559; doi: 10.1097/00005768-200203000-00025.

26. American College of Sports Medicine. American College of Sports Medicine position stand. Progression models in resistance training for healthy adults. Med Sci Sports Exerc. 2009;41(3):687-708; doi: 10.1249/ MSS.0b013e3181915670.

27. Cohen J. Statistical power analysis for the behavioral sciences. New York: Lawrence Erlbaum Associates; 1988.

28. Cronin J, McNair PJ, Marshall RN. Developing explosive power: a comparison of technique and training. $\mathrm{J}$ Sci Med Sport. 2001;4(1):59-70; doi: 10.1016/S14402440(01)80008-6.

29. Schenau GJ, Bobbert MF, de Haan A. Does elastic energy enhance work and efficiency in the stretch-shortening cycle? J Appl Biomech. 1997;13(4):389-415; doi: 10.1123/jab.13.4.389.

30. Goto K, Ishii N, Kizuka T, Kraemer RR, Honda Y, Takamatsu K. Hormonal and metabolic responses to slow movement resistance exercise with different durations of concentric and eccentric actions. Eur J Appl Physiol. 2009;106(5):731-739; doi: 10.1007/s00421009-1075-9.

31. Van den Tillaar R, Saeterbakken AH. Fatigue effects upon sticking region and electromyography in a six-repetition maximum bench press. J Sports Sci. 2013;31(16): 1823-1830; doi: 10.1080/02640414.2013.803593.
32. Kidgell DJ, Bonanno DR, Frazer AK, Howatson G, Pearce AJ. Corticospinal responses following strength training: a systematic review and meta-analysis. Eur J Neurosci. 2017;46(11):2648-2661; doi: 10.1111/ejn. 13710

33. Dutta A, Krishnan C, Kantak SS, Ranganathan R, Nitsche MA. Recurrence quantification analysis of surface electromyogram supports alterations in motor unit recruitment strategies by anodal transcranial direct current stimulation. Restor Neurol Neurosci. 2015;33(5):663-669; doi: 10.3233/RNN-140469.

34. Allen DG, Lamb GD, Westerblad H. Skeletal muscle fatigue: cellular mechanisms. Physiol Rev. 2008;88(1): 287-332; doi: 10.1152/physrev.00015.2007.

35. Jones DA, de Ruiter CJ, de Haan A. Change in contractile properties of human muscle in relationship to the loss of power and slowing of relaxation seen with fatigue. J Physiol. 2006;576(Pt 3):913-922; doi: 10.1113/ jphysiol.2006.116343.

36. Earp JE, Newton RU, Cormie P. Blazevich AJ. Inhomogeneous quadriceps femoris hypertrophy in response to strength and power training. Med Sci Sports Exerc. 2015;47(11):2389-2397; doi: 10.1249/MSS.0000000 000000669. 